\title{
Shot Noise Correlations in the Detection of Ultrashort Optical Pulses
}

\author{
F. Quinlan ${ }^{*}, 1$, T. M. Fortier ${ }^{1}$, H. Jiang ${ }^{1}$, A. Hati ${ }^{1}$, C. Nelson ${ }^{1}$, Y. Fu ${ }^{2}$, J. Campbell ${ }^{2}$, and S. A. Diddams ${ }^{\dagger, 1}$ \\ ${ }^{1}$ National Institute of Standards and Technology, Boulder, USA \\ ${ }^{2}$ Department of Electrical Engineering, University of Virginia, Charlottesville, VA \\ *fquinlan@,boulder.nist.gov, ${ }^{\dagger}$ sdiddams@,boulder.nist.gov
}

\begin{abstract}
The time-varying nature of optical pulses produces correlations in the photocurrent shot noise spectrum. These correlations are exploited to produce a phase noise floor of -177 $\mathrm{dBc} / \mathrm{Hz}$ on a $10 \mathrm{GHz}$ photonically generated microwave signal.
\end{abstract}

Keywords-microwave photonics; phase noise; shot noise; ultrafast optics

Many state-of-the-art photonic applications such as highspeed signal processing, time and frequency dissemination, and microwave signal generation rely on the ultralow pulse-to-pulse timing jitter of optical pulse trains from mode-locked lasers. The timing jitter of an optical pulse train can be assessed by photodetecting the pulse train and analyzing the phase noise of the photonically generated microwave signal. When locked to an optical frequency reference, the pulse timing jitter can be sub-femtosecond, producing microwave signals with spectral purity rivalling that of the best electronic oscillators [1].

The precision by which the phase noise of a photonically generated microwave signal is measured is ideally limited by shot noise. The conventional expression for the photocurrent variance due to shot noise assumes timeinvariant optical intensity, and therefore does not apply to the detection of optical pulse trains [2,3]. As first treated in the context of gravitational wave (GW) detectors, photodetection of a time-varying optical intensity produces correlations in the photocurrent shot noise spectrum [4]. These correlations were shown to give $\mathrm{a} \sim 1.8 \mathrm{~dB}$ increase of the detection noise when the GW photocurrent is demodulated. Here we show that these correlations can have a much more significant impact on the amplitude and phase noise of microwaves generated by the detection of ultrashort optical pulses. A semi-classical model of the shot noise is presented that predicts the shot noise contribution to the phase noise can be much lower than the contribution to the amplitude noise, depending on the optical pulse width. Preliminary measurements of the phase noise on a $10 \mathrm{GHz}$ photonically-derived microwave signal reveal a pulse width dependence to the shot noise level, with the

Financial support is provided by NIST. Contribution of an agency of the US government, not subject to copyright in the US.

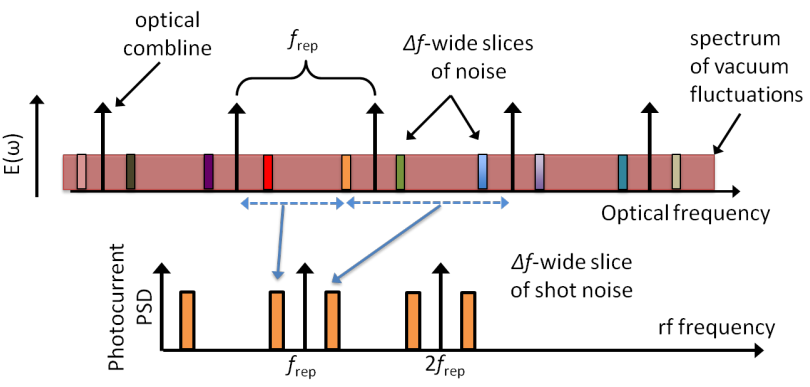

Fig. 1. Frequency domain picture of spectrum correlations in the photocurrent shot noise.

shortest optical pulses yielding a phase noise floor at -177 $\mathrm{dBc} / \mathrm{Hz}$. This is to our knowledge the lowest phase noise floor of a photonically generated microwave signal reported to date.

The shot noise imbalance between phase and amplitude noise can be understood with a frequency domain picture of the optical field. In the optical frequency domain, the pulse train is represented as a frequency comb where a fixed phase relationship exists among the comblines. Photocurrent shot noise is produced by the beating of the comblines with vacuum fluctuations [5]. The vacuum fluctuation field is represented as uncorrelated white noise in the optical domain. (Representing vacuum fluctuations as a classical noise is not strictly correct. However, this picture may be used as long as we exclude vacuum-vacuum beat noise in the photocurrent.) Consider a narrow slice of the noise in the optical domain beating with multiple comblines, as shown in the top of Fig. 1. Beating with different comblines translates this single narrow slice of noise to a multitude of rf frequencies. Since there is a fixed phase relationship among the comblines of the pulse train, these slices of photocurrent shot noise are correlated. Correlated noise exists symmetric about generated microwave carriers. It is the correlation of upper and lower noise sidebands about a microwave carrier that produces the imbalance of the carrier's amplitude and phase noise. This can be seen by considering the demodulation implicit in any amplitude or phase noise measurement. Demodulation folds the lower sideband onto the upper 
sideband, and, if they are correlated, the upper and lower sidebands interfere. Whether or not they interfere constructively of destructively depends on whether amplitude or phase noise is measured. The degree of correlation is a function of the number of comblines and their relative phases, and therefore the optical pulse width, since only the comblines at the ends of the spectrum contribute uncorrelated upper and lower noise sidebands about the microwave carrier. This noise fractionally decreases as the number of comblines increases.

To quantitatively derive the shot noise contribution to the amplitude and phase noise of a photonically generated microwave signal, the semi-classical approach to photodetection of [3] is used where the photocurrent $i(t)$ is the sum of elementary impulses given by $i(t)=\sum_{k} X_{k} h(t-k \Delta t)$. Here, $h(t)$ is the impulse response of the photodetector, and $X$ is a random variable equal to either 1 or 0 , depending on whether a photon is detected in time $\Delta t$. Phase and amplitude noise measurements are modeled by mixing the photocurrent with a noiseless reference, given by $\cos \left(2 \pi f_{r}+\Phi_{r}\right)$, and computing the resultant power spectral density. This results in a singlesideband noise-to-microwave power ratio of

$$
S=\frac{q I_{\text {avg }} R}{P_{r f}}[1 \pm C(\tau)]\left[H^{-1}\right]
$$

where $q$ is the fundamental charge, $I_{\text {avg }}$ is the average photocurrent, $R$ is the system impedance, $P_{r f}$ is the power of the photonically generated microwave signal at frequency $f_{r}, \tau$ is the optical pulse width, and $C(\tau)$ is the pulse width-dependent degree of correlation, ranging from $0\left(\tau \rightarrow 1 / f_{r}\right)$ to $1(\tau \rightarrow 0)$. In this expression, the plus sign is for amplitude noise, and the minus sign is for phase noise. The full expression for $C(\tau)$ is not given here, but for Gaussian shaped optical pulses $C(\tau)$ reduces to $\exp \left\{-\left(2 \pi f_{r} \tau\right)^{2}\right\}$. Fig. 2 shows the predicted noise on a 10 $\mathrm{GHz}$ carrier as a function of pulse width, assuming a train of Gaussian shaped optical pulses.

Experiments were performed to verify the predicted photocurrent shot noise behavior. The experimental setup consisted of a $2 \mathrm{GHz}$ repetition rate pulse train from a

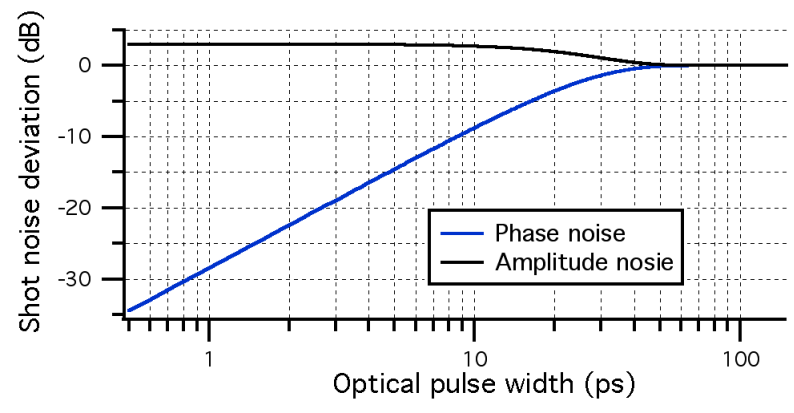

Fig. 2. Shot noise deviation from the long pulse limit as a function of optical pulse width. Amplitude and phase noise are shown for a $10 \mathrm{GHz}$ photonically generated microwave signal and Gaussian shaped pulses.
Ti:sapphire mode-locked laser illuminating a high speed photodiode. Phase noise measurements were performed at the $10 \mathrm{GHz}$ harmonic. The photodiode design is a modified uni-travelling carrier structure capable of producing high output saturation current [6]. This provided an $\sim 8 \mathrm{dBm} 10$ $\mathrm{GHz}$ signal, rendering rf amplification unneccesary and reduced the thermal noise floor below $-180 \mathrm{dBc} / \mathrm{Hz}$ at offset frequencies beyond $1 \mathrm{MHz}$. The width of the optical pulse illuminating the photodetector was varied by adding single mode fiber just before the detector. The $10 \mathrm{GHz}$ harmonic was isolated with a narrow bandpass filter and mixed in quadrature with an ultralow phase noise reference oscillator. The focus of our phase noise measurements was on offset frequencies of $1 \mathrm{MHz}-10 \mathrm{MHz}$, as this range is free from technical noise from the mode-locked laser. A 1 $\mathrm{kHz}$ bandwidth phase lock between the $10 \mathrm{GHz}$ harmonic from the photodetector and the microwave phase reference was employed to maintain phase quadrature during measurement. The low lock bandwidth had negligible effect for offset frequencies past $1 \mathrm{MHz}$. Fig. 3 shows phase noise measurements for 3 ps and 14 ps pulse widths. The shortest optical pulse produced a phase noise floor at -177 $\mathrm{dBc} / \mathrm{Hz}$. From the assumptions of the shot noise model presented here, this noise floor is not believed to be shot noise limited. The reason for this floor is currently under investigation.

In conclusion, shot noise corelations resulting from the detection of optical pulses can be used to tremendous advantage to reduce the phase noise floor of photonically derived microwaves. Although here we have focused on shot noise, any periodic noise, such as signal-spontaneous beat noise from optical amplification, should display the same behavior.

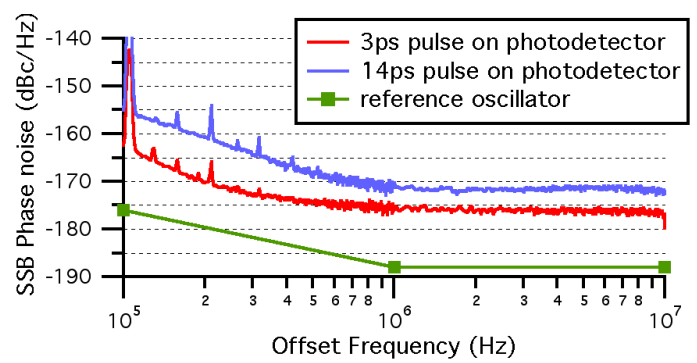

Fig. 3. Single sideband (SSB) phase noise measurements for $3 \mathrm{ps}$ and $14 \mathrm{ps}$ optical pulses. The noise from $1 \mathrm{MHz}$ to $10 \mathrm{MHz}$ decreases for shorter pulses as a consequence of shot noise correlations. Below $1 \mathrm{MHz}$, the difference in the noise curves in attributed to noise on the pulse shape.

\section{REFERENCES}

[1] T. Fortier, et al., Nat. Photon., 5, p.425 (2011)

[2] P. Winzer, JOSA B, 14, p.2424 (1997)

[3] B. E. A. Saleh \& M. C. Teich, Fundamentals of Photonics, Wiley, New York, 1991

[4] T. M. Niebauer et al., Phys. Rev. A, 43, p.5022 (1991)

[5] H. A. Bachor \& P. J. Manson, J. Mod. Opt., 37, p.1727 (1990)

[6] Z. Li et al., IEEE JQE, 46, p.626 (2010) 